\title{
Effects of Post-Activation Potentiation (PAP) Warm-up on Vertical Jump Performance among Volleyball Players
}

\author{
Diong Zhi Ling \\ Faculty of Educational Studies, Universiti Putra Malaysia \\ 43400 UPM Serdang, Selangor, Malaysia \\ Chee Chen Soon (Corresponding Author) \\ Faculty of Educational Studies, Universiti Putra Malaysia \\ 43400 UPM Serdang, Selangor, Malaysia \\ Mohd Rozilee Wazir Norjali Wazir \\ Faculty of Educational Studies, Universiti Putra Malaysia \\ 43400 UPM Serdang, Selangor, Malaysia
}

\begin{abstract}
Kok Lian Yee
Faculty of Applied Sciences, Tunku Abdul Rahman Universiti Kolej

53300 Kuala Lumpur, Wilayah Persekutuan Kuala Lumpur, Malaysia
\end{abstract}

Received: Oct. 8, $2021 \quad$ Accepted: Nov. 15, $2021 \quad$ Online published: Nov. 25, 2021

doi:10.5296/ijhrs.v11i4S.19248 URL: https://doi.org/10.5296/ijhrs.v11i4S.19248

\begin{abstract}
This study aims to examine the effects of resistance versus complex exercise with the retention of post-activation potentiation (PAP) on vertical jump among volleyball players. A total of 30 volleyball players (mean age $=16.5 \pm 1.106$ years old) were recruited as participants of this study. The participants were requested to attend two different testing sessions, with two days between each session. Each session had a different exercise routine,
\end{abstract}


namely, resistance and complex. A one-way repeated measure was used to analyse the performance of the vertical jump, while a pairwise comparison was applied to compare the retention of PAP between the resistance and complex exercises. The findings show that there is a significant interaction for both warm-up exercises; $[(\mathrm{F}(1,29)=1275.57, \mathrm{p}=0.000]$ for resistance exercise and $\left[\left(\mathrm{F}_{1,29}\right)=1456.83, \mathrm{p}=0.000\right]$ for complex exercise. The retention analysis for PAP effect also indicates that there is a significant difference between the two warm-up activities in vertical jump performance. In conclusion, this study reveals that both exercises managed to increase PAP among volleyball players. However, complex exercise is suggested to be combined with the warm-up method as it can maintain a longer retention than resistance exercise.

Keywords: Post-activation potentiation (PAP), resistance exercise, complex exercise, vertical jump, volleyball players

\section{Introduction}

Warm-up has become a common practice before any physical activity is performed (Wood et al., 2007) and its purpose is to prepare the body mentally and physically. There are also warm-up methods used to procure post-activation potentiation (PAP) effects. PAP has been shown to be able to increase maximum strength and acute power during training or competition (Hodgson et al., 2005; Mcbride et al., 2005). PAP also refers to a neuromuscular phenomenon that tends to increase power after stimulation of the contractile. PAP stimulation is associated with two major interaction mechanisms, namely increased light chain phosphorylation and increased motor unit recruitment (increased neural drive).

PAP effects may be difficult or less sustainable without resistance exercise through warm-up methods. Many previous research have been conducted in relation to PAP in various sports, such as rugby (Mitchell \& Sale, 2011), athletics (Crewther et al., 2011), and football (Mola et al., 2014). Nevertheless, the findings have always sparked controversy because of the different subjects, such as physical level, training experience, training background, and sports. Apart from that, the variable, e.g. the intensity of the exercise, could be one of the factors that affect PAP. According to Sale (2002) and Gilbert et al. (2005), high-intensity exercise appears to be more effective on PAP. Despite this fact, there is still a lack of studies on the effect of PAP between resistance and complex exercises as a warm-up method on the performance of vertical jump in volleyball sports.

Besides, previous studies showed that the retention of PAP effect with resistance exercise can increase power between 2 to 20 minutes period (Sue et al., 2016). While findings by Bauer et al. (2019) revealed that the effect of PAP could only be maintained for 10 minutes and did not exceed 30 minutes (Wilson et al., 2013). A study by Sanchez et al. (2018) suggested that the effects of PAP could be studied using physical fitness tests by repeating the same test session. Therefore, the present research was conducted to determine the effect of PAP between resistance and complex exercises, and the retention of vertical jump performance for 30 minutes, in order to increase power.

The most determinant performance factor in volleyball is power during the vertical jump. The 


\section{Macrothink}

International Journal of Human Resource Studies

ISSN 2162-3058

2021, Vol. 11, No. 4S

effectiveness of vertical jump allows for obtaining improvements in technical actions, such as sets, hits, services, or blocks to achieve success in a volleyball game. Therefore, the purpose of this study is to examine the effect of PAP between resistance and complex exercises and the retention on vertical jump among volleyball players.

\section{Method}

\section{Research design}

A one-way repeated measure design (two types of warm-up exercises $\mathrm{x}$ seven trials) together with a crossover design to control order effect of the warm-up exercises was utilised, whereby each subject needed to perform both resistance and complex exercises.

\section{Participants}

A total of 30 volleyball players were recruited from the Hin Hua High School, in which (age $=16.5 \pm 1.106$ years, height $=173.60 \pm 5.55 \mathrm{~cm}$, and body mass $=59.56 \pm 3.79 \mathrm{~kg}$.). The participants had to be physically active in volleyball training for two years and represented the district or state in 2019. All participants also underwent training for at least five days a week with a training session from 3.30 p.m. to 6.30 p.m. The participants had to be free of injury or any medicine, which might influence their performance.

Table 1. Descriptive information for participants

\begin{tabular}{ccccc}
\hline $\boldsymbol{N}$ & Age (years) & Height(cm) & Weight(kg) & 6-RM BS \\
\hline 30 & $16.5 \pm 1.106$ & $173.60 \pm 5.55$ & $59.56 \pm 3.79$ & $58.58 \pm 3.62$ \\
\hline
\end{tabular}

*Participant means and standard deviations for descriptive information;

BS - Back Squat; 6-RM - six repetition maximum

\section{Procedures}

Session 1 consisted of a one-hour study orientation to explain the objective and procedure of the research. The researcher recorded the participants' age, height, weight, and 6-RM. The researcher then showed the technique for back squat, standing jump and reach, and vertical jump to the participants. The 6-RM back squat was assessed as described below after the orientation. 6-RM back squat as the resistance exercise and 6-RM back squat followed by six times standing jump and reach as the complex exercise were carried out during Sessions 2 and 3 to induce PAP. 


\begin{tabular}{|c|c|c|}
\hline $\begin{array}{c}\text { Session 1 } \\
\text { Session 1: Orientation }\end{array}$ & $\begin{array}{c}\text { Session } 2 \\
\text { Pre-test: Vertical Jump }\end{array}$ & $\begin{array}{c}\text { Session } 3 \\
\text { Pre-test: Vertical Jump }\end{array}$ \\
\hline $\begin{array}{c}\text { Record Age, Height and } \\
\text { Weight }\end{array}$ & $\begin{array}{l}\text { Group A: Resistance Exercise } \\
\text { Group B: Complex Exercise }\end{array}$ & $\begin{array}{l}\text { Group A: Complex Exercise } \\
\text { Group B: Resistance Exercise }\end{array}$ \\
\hline Collect 6-RM Back Squat & $\begin{array}{l}\text { Post-test: } 15 \text { seconds, } 5,10,15,20 \\
\text { and } 30 \text { minutes }\end{array}$ & $\begin{array}{c}\text { Post-test: } 15 \text { seconds, } 5,10,15,20 \\
\text { and } 30 \text { minutes }\end{array}$ \\
\hline
\end{tabular}

Figure 1. PAP retention overview of warm-ups. PAP: post-activation potentiation; 6-RM back squat as resistance exercise warm-up; 6-RM followed by standing jump and reach as complex exercise warm-up

\section{Vertical jump test (VJ)}

The participants were given a vertical jump pre-test before performing the warm-up exercises. The post-tests were recorded at post-15 seconds, post-5 minutes, post-10 minutes, post- 15 minutes, post-20 minutes, and post-30 minutes after performing the warm-up exercises. The height of the jump was determined by the vertec vanes shot and recorded in $\mathrm{cm}$ units. In each of the post-tests, the participants were given two attempts to jump.

\section{Resistance exercise}

The participants performed back squat that involved quadriceps muscle, biceps muscle, and spinal erector. They were asked to perform one set of 6-RM back squat as an experimental session.

\section{Complex exercise}

In this study, complex exercise is a combination of resistance exercise and plyometric exercise. The participants performed one set of 6-RM back squat followed by standing jump and reach. Rest between exercises was 30 seconds.

\section{Results}

Based on the findings, the mean and standard deviation in the vertical jump scores for the resistance exercise were as follows: 15 seconds post-test $(\mathrm{M}=55.83, \mathrm{SD}=8.61), 5$ minutes post-test $(\mathrm{M}=56.34, \mathrm{SD}=8.60), 10$ minutes post-test $(\mathrm{M}=55.93, \mathrm{SD}=8.65), 15$ minutes post-test $(\mathrm{M}=55.60, \mathrm{SD}=8.56), 20$ minutes post-test $(\mathrm{M}=55.30, \mathrm{SD}=8.51)$, and 30 minutes post-test $(\mathrm{M}=55.04, \mathrm{SD}=8.44)$ as compared to pre-test $(\mathrm{M}=54.65, \mathrm{SD}=8.30)$. There was an increase in the mean vertical jump score for the resistance exercise by $2.18 \%, 3.09 \%, 2.34 \%, 1.74 \%$, $1.19 \%$, and $0.71 \%$ for 15 seconds, $5,10,15,20$, and 30 minutes post-tests, respectively, as compared to pre-test. In contrast, the mean and standard deviation in the vertical jump score for the complex exercise were recorded as: 15 seconds post-test $(\mathrm{M}=55.95, \mathrm{SD}=8.24), 5$ minutes post-test $(\mathrm{M}=56.99, \mathrm{SD}=8.25), 10$ minutes post-test $(\mathrm{M}=57.47, \mathrm{SD}=8.42), 15$ minutes post-test $(\mathrm{M}=57.94, \mathrm{SD}=8.06), 20$ minutes post-test $(\mathrm{M}=58.59, \mathrm{SD}=8.29)$, and 30 minutes post-test $(\mathrm{M}=57.87, \mathrm{SD}=7.97)$ as compared to pre-test $(\mathrm{M}=54.75, \mathrm{SD}=8.40)$. There was an 
increase in the mean vertical jump score for the complex exercise by $2.20 \%, 4.09 \%, 4.97 \%$, $6.02 \%, 7.01 \%$, and $5.70 \%$ for 15 seconds, 5, 10, 15, 20 and 30 minutes post-tests, respectively, as compared to pre-test. The mean score for complex exercise was higher than the mean score of the resistance exercise.

Table 2. Participants means (M) and standard deviations (SD) for descriptive information; *Complex Exercise were significantly greater than Resistance Exercise $(\mathrm{p}<.05)$. Paired t-test p-values

\begin{tabular}{lccccccc}
\hline & Pre-test & & \multicolumn{2}{c}{ Post-test } \\
& & $\mathbf{1 5}$ seconds & $\mathbf{5}$ minutes & $\mathbf{1 0}$ minutes & $\mathbf{1 5}$ minutes & $\mathbf{2 0}$ minutes & $\mathbf{3 0}$ \\
minutes
\end{tabular}

Based on the data, it showed a significant effect for warm-up activities $(p<0.01)$ at post-5, 10, 15, 20, and 30 minutes. Repeated ANOVA that measured VJ indicated a slight significance at time point following the resistance exercise $(p<0.05)$ and reached peak time at 5 minutes, whereas VJ following the complex exercise varied significantly over time $(p<$ 0.01) and reached peak time at 20 minutes. Post-hoc techniques (paired t-tests) determined that VJ following the complex exercise was significantly greater at $5,10,15,20$, and 30 minutes as compared to the VJ scores following the resistance exercise $(p<0.05)$. However, there was no significance at 15 seconds and 5 minutes $(p>0.05)$. The results of the pair $t$-tests were as follows: pre-test: $p=0.153,15$ seconds: $p=0.483,5 \min : p=0.009,10$ min: $p=0.000,15 \min : p=0.000,20 \min : p=0.000$, and $30 \min : p=0.000$.

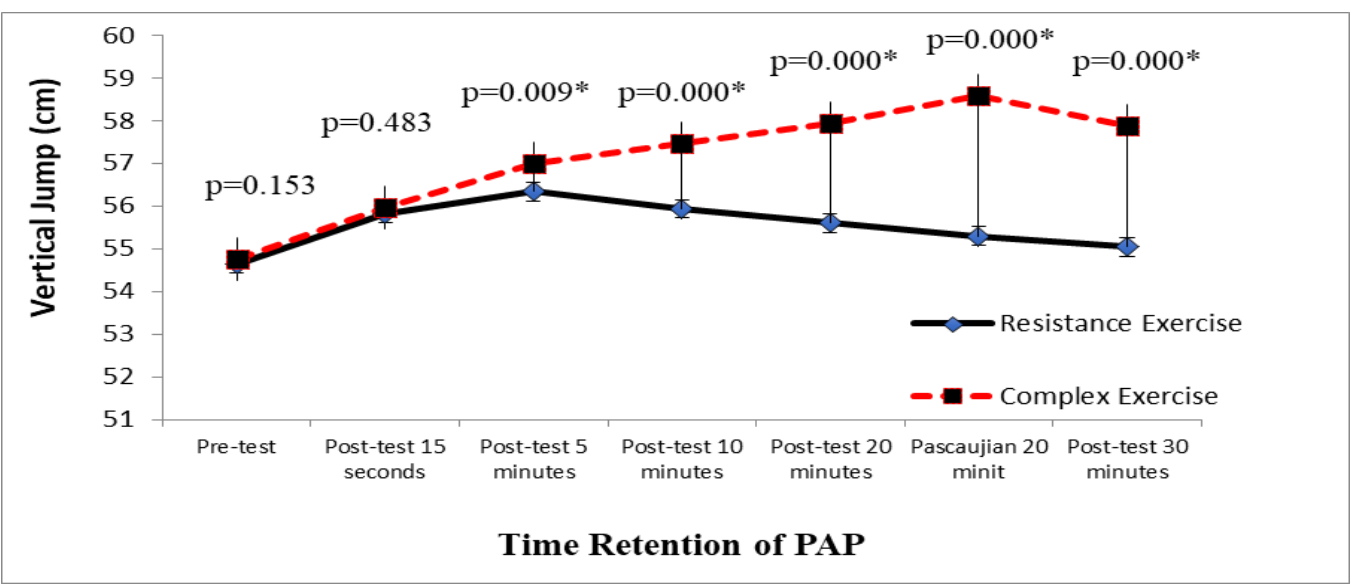

Graf 1. A pairwise comparison of the duration of retention of the PAP effect between resistance exercise and complex exercise

\section{Discussion}

This study was conducted to examine the effect of PAP on vertical jump among volleyball 
players. The effect of PAP plays an important role in the warm-up as it can help to improve performance in training or competition. The objective of this study is to determine that the warm-up activity with a single 6-RM set of the back squat is effective at inducing PAP and increasing the performance of $\mathrm{VJ}$ and the duration of retention. The data for the resistance exercise suggested that warm-up with a single 6-RM set of the back squat as the resistance exercise induced PAP and increased VJ for up to 5 minutes. VJ increased for the first two time points, which were 15 seconds and 5 minutes before showing a decrease, suggesting that the optimal retention of PAP for the resistance exercise's time point was around 5 minutes. However, the complex exercise indicated that a single 6-RM set of the back squat followed by six continuous standing jump and reach induced PAP and increased VJ for up to 20 minutes. VJ increased in the first five before decreasing, suggesting that the optimal retention of PAP for the complex exercise was around 20 minutes. The findings of this study were consistent with the previous research that complex exercise managed to improve effectiveness of the leg power when combined with warm-up activities (Carter et al., 2014) as compared to resistance exercise and the retention of PAP. Based on the previous studies related to the comparison of complex exercise, plyometric exercise, and resistance exercise on the effects of PAP in judo and taekwondo, Miarka et al. (2011) showed that there was a significant increase in judo performance after performing the plyometric exercise with ten sets of three jumps consecutively, but did not show distant improvement between the resistance exercise with five sets of 95\% 1-RM and complex exercise that combined three sets of two repetitions of 90\% 1-RM with five consecutive jumps. Conversely, a study by Da Santos et al. (2015) revealed a significant improvement in taekwondo after a complex exercise by combining three sets of two repetitions of 95\% 1-RM half squat repetitions with four vertical jumps, but no significant improvement after the resistance exercise (three sets of 95\% 1-RM half squat repetitions) and plyometric exercise (three sets of ten repetitions of vertical jumps). It can be concluded that the inconsistencies of the research by Miarka et al. (2011) and Da Silva Santos et al. (2015) with this study were related to the different protocols used to stimulate PAP, such as intensity, volume, type of conditioning exercise, and recovery time. Lack of improvement in VJ performance during the resistance and complex exercises may be explained by the individual phenomenon of PAP (Comyns et al., 2010). For example, the study by Chen et al. (2017) indicated that participant 9 showed the greatest improvement in the resistance exercise, while participant 10 had the greatest improvement in the complex exercise. In addition, Bevan et al. (2010), Esformes et al. (2013), Fukutani et al. (2014), and Wilson et al. (2013) stated that the effect of PAP exists inconsistently to various factors influencing activity phenomena, such as activity type, intensity, volume, strength and power level, training background, rest time between warm-up activities, and subsequent activities. Robbins et al. (2005) reported that an individual's PAP response was affected by muscular strength, type of fibre, training level, experience in sport, and gender, all of which have not been identified. Previous investigations stated that single conditioning exercises showed different individual PAP responses due to conditioning levels. Researchers have suggested that coaches should evaluate the athletes before assigning a single conditioning exercise as part of their warm-up routine (Lim et al., 2013; Bogdanis et al., 2014). In summary, at least for trained subjects, the data of this study indicate that there are no recommendations for 
optimal activity content such as volume and intensity for obtaining PAP effects. Furthermore, because it is possible for stronger athletes to benefit from participating in adaptive activities in their warm-up routines, coaches are encouraged to always evaluate athletes' responses to different warm-up activities' protocols by adjusting or changing the training content when necessary. Of course, both of these activities can improve an athlete's performance in many types of sports, especially sports that rely on leg power. This study was limited by the lack of individuals' rest periods. As shown by Gołaś et al. (2016), the individualisation of rest periods after load-related warm-up activities is important to find the best strategy for each athlete for different sports. Therefore, the findings in this study proved that the manipulation of variables, i.e. 6-RM in one set of resistance and complex exercises, could improve the leg power among volleyball players.

\section{Acknowledgments}

The authors would like to express their deepest gratitude to the respondents who were willing to participate and provide full cooperation in this study.

\section{References}

Bauer, P., Sansone, P., Mitter, B., Makivic, B., Seitz, L. B., \& Tschan, H. (2019). Acute Effects of Back Squats on Countermovement Jump Performance Across Multiple Sets of a Contrast Training Protocol in Resistance-Trained Men. Journal of Strength and Conditioning Research, 33(4), 995-1000. https://doi.org/10.1519/JSC.0000000000002422

Bevan, H. R., Cunningham, D. J., Tooley, E. P., Owen, N. J., Cook, C. J., \& Kilduff, L. P. (2010). Influence of Postactivation Potentiation on Sprinting Performance in Professional Rugby Players. Journal of Strength and Conditioning Research, 24(3), 701-705. https://doi.org/10.1519/JSC.0b013e3181c7b68a

Bogdanis, G. C., Tsoukos, A., Veligekas, P., Tsolakis, C., \& Terzis, G. (2014). Effects of Muscle Action Type With Equal Impulse of Conditioning Activity on Postactivation Potentiation. Journal of Strength and Conditioning Research, 28(9), 2521-2528. https://doi.org/10.1519/JSC.0000000000000444

Carter, J., \& Greenwood, M. (2014). Complex Training Reexamined. Strength \& Conditioning Journal, 36(2), 11-19. https://doi.org/10.1519/SSC.0000000000000036

Chen, Z., Lo, S., Wang, M., Yu, C., \& Peng, H. (2017). Can Different Complex Training Improve the Individual Phenomenon of Post-Activation Potentiation? Journal of Human Kinetics, 56(1), 167-175. https://doi.org/10.1515/hukin-2017-0034

Comyns, T. M., Harrison, A. J., \& Hennessy, L. K. (2010). Effect of Squatting on Sprinting Performance and Repeated Exposure to Complex Training in Male Rugby Players. Journal of Strength and Conditioning Research, 24(3), 610-618. https://doi.org/10.1519/JSC.0b013e3181c7c3fc

Crewther, B. T., Kilduff, L. P., Cook, C. J., Middleton, M. K., Bunce, P. J., \& Yang, G. (2011). The Acute Potentiating Effects of Back Squats on Athlete Performance. Journal of Strength 
Chen, Z., Lo, S., Wang, M., Yu, C., \& Peng, H. (2017). Can Different Complex Training Improve the Individual Phenomenon of Post-Activation Potentiation? Journal of Human Kinetics, 56(1), 167-175. https://doi.org/10.1515/hukin-2017-0034

Comyns, T. M., Harrison, A. J., \& Hennessy, L. K. (2010). Effect of Squatting on Sprinting Performance and Repeated Exposure to Complex Training in Male Rugby Players. Journal of Strength and Conditioning Research, 24(3), 610-618. https://doi.org/10.1519/JSC.0b013e3181c7c3fc

Crewther, B. T., Kilduff, L. P., Cook, C. J., Middleton, M. K., Bunce, P. J., \& Yang, G. (2011). The Acute Potentiating Effects of Back Squats on Athlete Performance. Journal of Strength and Conditioning Research, 3319-3325. https://doi.org/10.1519/JSC.0b013e318215f560

Esformes, J. I., \& Bampouras, T. M. (2013). Effect of Back Squat Depth on Lower-Body Postactivation Potentiation. Journal of Strength and Conditioning Research, 27(11), 2997-3000. https://doi.org/10.1519/JSC.0b013e31828d4465

Fukutani, A., Takei, S., Hirata, K., Miyamoto, N., Kanehisa, H., \& Kawakami, Y. (2014). Influence of the Intensity of Squat Exercises on the Subsequent Jump Performance. Journal of Strength and Conditioning Research, 28(8), 2236-2243. https://doi.org/10.1519/JSC.0000000000000409

Gilbert, G., \& Lees, A. (2005). Changes in the force development characteristics of muscle following repeated maximum force and power exercise. Ergonomics, 48(11-14), 1576-1584. https://doi.org/10.1080/00140130500101163

Gołaś, A., Maszczyk, A., Zajac, A., Mikołajec, K., \& Stastny, P. (2016). Optimizing post activation potentiation for explosive activities in competitive sports. Journal of Human Kinetics, 52(1), 95-106. https://doi.org/10.1515/hukin-2015-0197

Hodgson, M., Docherty, D., \& Robbins, D. (2005). Post-Activation Potentiation. Sports Medicine, 35(7), 585-595. https://doi.org/10.2165/00007256-200535070-00004

Lim, J. J., \& Kong, P. W. (2013). Effects of Isometric and Dynamic Postactivation Potentiation Protocols on Maximal Sprint Performance. Journal of Strength and Conditioning Research, 27(10), 2730-2736. https://doi.org/10.1519/JSC.0b013e3182815995

Mcbride, J. M., Nimphius, S., \& Erickson, T. M. (2005). The Acute Effects Of Heavy-Load Squats And Loaded Countermovement Jumps On Sprint Performance. Journal of Strength $\begin{array}{lll}\text { and Conditioning } & \text { Research, } & \text { 893-897. }\end{array}$ https://doi.org/10.1519/00124278-200511000-00029

Miarka, B., Vecchio, F. B., \& Franchini, E. (2011). Acute Effects and Postactivation Potentiation in the Special Judo Fitness Test. Journal of Strength and Conditioning Research, 25(2), 427-431. https://doi.org/10.1519/JSC.0b013e3181bf43ff 
Mitchell, C. J., \& Sale, D. G. (2011). Enhancement of jump performance after a 5-RM squat is associated with postactivation potentiation. European Journal of Applied Physiology, 111(8), 1957-1963. https://doi.org/10.1007/s00421-010-1823-x

Mola, J. N., Bruce-Low, S. S., \& Burnet, S. J. (2014). Optimal Recovery Time for Postactivation Potentiation in Professional Soccer Players. Journal of Strength and Conditioning Research, 28(6), 1529-1537. https://doi.org/10.1519/JSC.0000000000000313

Robbins, D. W., \& Docherty, D. (2005). Effect Of Loading On Enhancement Of Power Performance Over Three Consecutive Trials. Journal of Strength and Conditioning Research, 19(4), 898-902. https://doi.org/10.1519/00124278-200511000-00030

Sale, D. G. (2002). Postactivation Potentiation: Role in Human Performance. Exercise and Sport Sciences Reviews, 30(3), 138-143. https://doi.org/10.1097/00003677-200207000-00008

Sanchez-Sanchez, J., Rodriguez, A., Petisco, C., Ramirez-Campillo, R., Martínez, C., \& Nakamura, F. Y. (2018). Effects of Different Post-Activation Potentiation Warm-Ups on Repeated Sprint Ability in Soccer Players from Different Competitive Levels. Journal of Human Kinetics, 61(1), 189-197. https://doi.org/10.1515/hukin-2017-0131

Santos, J. F., Valenzuela, T. H., \& Franchini, E. (2015). Can Different Conditioning Activities and Rest Intervals Affect the Acute Performance of Taekwondo Turning Kick? Journal of $\begin{array}{llll}\text { Strength } \quad \text { Conditioning 1640-1647. } & \text { Research, 29(6), }\end{array}$ https://doi.org/10.1519/JSC.0000000000000808

Sue, R. A., Adams, K., \& Debeliso, M. (2016). Optimal Timing for Post-Activation Potentiation in Women Collegiate Volleyball Players. Sports, 4(2), 27. https://doi.org/10.3390/sports4020027

Till, K. A., \& Cooke, C. (2009). The Effects of Postactivation Potentiation on Sprint and Jump Performance of Male Academy Soccer Players. Journal of Strength and Conditioning Research, 23(7), 1960-1967. https://doi.org/10.1519/JSC.0b013e3181b8666e

Wilson, J. M., Duncan, N. M., Marin, P. J., Brown, L. E., Loenneke, J. P., Wilson, S. M., . . . Ugrinowitsch, C. (2013). Meta-Analysis of Postactivation Potentiation and Power. Journal of

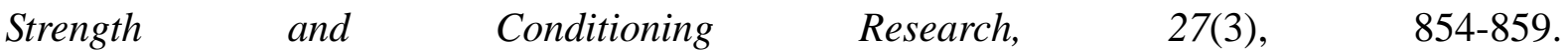
https://doi.org/10.1519/JSC.0b013e31825c2bdb

Woods, K., Bishop, P., \& Jones, E. (2007). Warm-Up and Stretching in the Prevention of

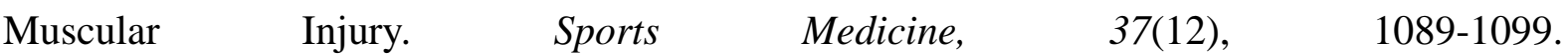
https://doi.org/10.2165/00007256-200737120-00006

\section{Copyright Disclaimer}

Copyright for this article is retained by the author(s), with first publication rights granted to the journal.

This is an open-access article distributed under the terms and conditions of the Creative Commons Attribution license (http://creativecommons.org/licenses/by/4.0/). 